\title{
MicroRNA-93 is overexpressed and induces apoptosis in glaucoma trabecular meshwork cells
}

\author{
YANSA WANG, FENGHUA LI and SHUYUN WANG \\ Department of Ophthalmology, Linyi People's Hospital, Linyi, Shandong 276000, P.R. China \\ Received September 22, 2015; Accepted October 3, 2016
}

DOI: $10.3892 / \mathrm{mmr} .2016 .5938$

\begin{abstract}
Glaucoma is an optic neuropathy and a major cause of blindness globally. Trabecular meshwork cells are important in maintaining aqueous humor flow, the dysfunction of which tends to induce glaucoma. As important regulators of gene expression, microRNAs may be crucial in regulating trabecular meshwork cells. The present study aimed to reveal the effect of microRNA-93 (miR-93) on glaucoma trabecular meshwork (GTM) cell apoptosis. The expression levels of miR-93 were compared between human trabecular meshwork (HTM) cells and GTM cells. The expression of miR-93 was inhibited and increased by transfecting the cells with a lentivirus containing its specific inhibitor sponge and expression vector to investigate changes in GTM cell viability and apoptosis. Alterations in the protein expression of nuclear factor erythroid 2-like 2 (NFE2L2) were also examined to elucidate the possible mechanism underlying the effects of miR-93. The results showed a marked promotion in the expression of miR-93 in the GTM cells, compared with the HTM cells $(\mathrm{P}<0.01)$. GTM cell viability was increased and its apoptosis was inhibited by transfection with the miR-93 sponge $(\mathrm{P}<0.01$ and $\mathrm{P}<0.001$, respectively), whereas the overexpression of miR-93 abrogated these effects $(\mathrm{P}<0.05$ and $\mathrm{P}<0.0001$, respectively). The expression of NFE2L2, a possible target of miR-93, was promoted by transfection with the miR-93 sponge $(\mathrm{P}<0.01)$ and was inhibited in the cells overexpressing miR-93 $(\mathrm{P}<0.01)$. Therefore, miR-93 was capable of inhibiting viability and inducing apoptosis of the GTM cells, which was achieved via the suppression of NFE2L2. These results elucidated the pro-apoptotic effects of miR-93 in GTM cells and its possible functional mechanism, providing potential therapeutic targets for the treatment of glaucoma.
\end{abstract}

Correspondence to: Dr Shuyun Wang, Department of Ophthalmology, Linyi People's Hospital, 27 Jiefang Road, Linyi, Shandong 276000, P.R. China

E-mail: wangshuyun69_29@126.com

Key words: glaucoma, trabecular meshwork cell, microRNA-93, apoptosis, nuclear factor erythroid 2-like 2

\section{Introduction}

Glaucoma is an optic neuropathy, which has typical structural and functional defects, and has long been the second leading cause of blindness after cataracts (1), with almost 60,000,000 individuals affected and 8,400,000 with bilateral blindness worldwide in 1979 (2). As a result of its asymptomatic and chronic characteristics, glaucoma is difficult to diagnose at an earlier stage. Glaucoma is divided into two major forms: Open-angle glaucoma and angle-closure glaucoma, which are associated with the death of a substantial number of retinal ganglion cells in the inner retina and the loss of their axons in the optic nerve (2). Glaucoma is considered to be induced by an increase in intraocular pressure. Trabecular meshwork cells can regulate aqueous humor outflow and control intraocular pressure (3), however, the degradation of these cells, for example in primary open-angle glaucoma, leads to the loss of aqueous humor drainage and results in a consequent increase in intraocular pressure $(4,5)$. Therefore, preventing the loss of trabecular meshwork cells is pivotal for controlling glaucoma.

MicroRNAs (miRNAs) are small non-coding RNA molecules, which guide argonaute proteins to their target RNAs and modulate gene expression post-transcriptionally (6). They are generated from transcription by RNA polymerase and are cleaved by ribonucleases, Drosha and Dicer, from primary miRNA into precursor miRNA, and then to mature miRNA (7). The regulatory mechanism of miRNAs may vary among different target RNAs, cells and conditions (8). miRNA regulation is important in the altered gene expression, which occurs in disease (9), and may be more effective than gene methylation or histone modification (10). miRNAs are vital in trabecular meshwork cell regulation, as revealed in previous studies. A previous study showed that miR-200c significantly decreases intraocular pressure via regulation of its targets; including Zinc finger E-box binding homeobox 1 and 2 (11). Several miRNAs have been shown to be aberrantly expressed in human trabecular meshwork (HTM) cells with stress-induced premature senescence (12), of which miR-183 regulates integrin $\beta 1$ and thus modulating the senescence of HTM cells (13). Despite these previous studies involving miRNAs, the association between miRNAs and HTM cell proliferation and apoptosis remains to be elucidated.

miR-93 has been investigated in human colon cancer stem cells and non-small cell lung cancer cells. It is downregulated 
in the former cells, with its overexpression leading to inhibited cell proliferation and colony formation (14), whereas the overexpression of miR-93 in the latter cells inhibits the expression of tumor suppressor candidate 2 (15). In this context, the present study hypothesized that miR-93 is involved in the regulation of glaucoma trabecular meshwork (GTM) cell apoptosis. To elucidate the role of miR-93 in GTM cell apoptosis, a lentivirus containing the miR-93-specific inhibitor sponge and expression vector were transfected into GTM cells to monitor the effects on cell viability and apoptosis. In addition, the expression of a possible associated factor in these processes was examined to determine the functional mechanism of miR-93. The results of these investigations may provide a basic understanding of the role of miR-93 in regulating GTM cells.

\section{Materials and methods}

Cells. The HTM and GTM cells were provided by Zhongshan Ophthalmic Center of Sun Yat-sen University (Guangzhou, China). The cells were cultured in Dulbecco's modified Eagle's medium (DMEM)/F12 supplemented with $20 \%$ fetal bovine serum in a humidified atmosphere with $5 \% \mathrm{CO}_{2}$ at $37^{\circ} \mathrm{C}$. The cells were passaged when their confluence reached $70 \%$ and were washed twice with D-Hanks solution.

Lentivirus transfection. The lentiviral vectors specific for pre-miR-93 and miR-93 sponge were constructed by GenePharma (Shanghai, China). Prior to transfection, the GTM cells were transferred to 24 -well plates $\left(1 \times 10^{5}\right.$ cells/well $)$ and cultured for $24 \mathrm{~h}$. The lentivirus suspension was added to the cells using Lipofectamine 3000 (Invitrogen; Thermo Fisher Scientific, Inc., Waltham, MA, USA) according to the manufacturer's protocol, and blank lentivirus was used in the control group. Following incubation for $24 \mathrm{~h}$ at $37^{\circ} \mathrm{C}$, the suspension in the wells was replaced with DMEM/F12 medium and the cells were cultured for another $48 \mathrm{~h}$ at $37^{\circ} \mathrm{C}$. The transfection efficiency was monitored using a fluorescence-activated cell sorter (BD FACSCanto II; BD Biosciences, San Jose, CA, USA), and the cells were collected for further analyses.

Cell viability assay. The transfected GTM cells were adjusted to the concentration of $5 \times 10^{4} / \mathrm{ml}$, transferred to 96 -well plates (200 $\mu \mathrm{l}$ per well), and cultured at $37^{\circ} \mathrm{C}$ for $24,48,72$ and $96 \mathrm{~h}$. Fresh medium containing $0.5 \mathrm{mg} / \mathrm{ml}$ methyl thiazolyl tetrazolium (Sigma-Aldrich; Merck Millipore, Darmstadt, Germany) was added to each well and the cells were incubated at $37^{\circ} \mathrm{C}$ for $4 \mathrm{~h}$, following which the reaction was terminated using $200 \mu 1$ dimethyl sulfoxide. The optical density at $570 \mathrm{~nm}$ was detected for each well using a microplate reader (Molecular Devices LLC, Silicon Valley, CA, USA).

Cell apoptosis assay. The transfected GTM cells were transferred to 24 -well plates $48 \mathrm{~h}$ following transfection, at a density of $2 \times 10^{5}$ cells per well. Cell apoptosis was analyzed using the annexin V-fluorescein isothiocyanate (FITC)/prodium iodide (PI) dual staining method with an Annexin V:FITC Apoptosis Detection kit II (BD Biosciences) according to the manufacturer's protocol. The cells were then analyzed using the BD FACSCanto II. The percentages of apoptotic cells (annexin V positive and PI negative) were compared.
Reverse transcription-quantitative polymerase chain reaction (RT-qPCR) analysis. The microRNAs of the HTM and GTM cells were extracted using RNAiso for small RNAs (Takara Biotechnology Co., Ltd., Dalina, China) and reverse transcribed using a One Step PrimeScript miRNA cDNA synthesis kit (Takara Biotechnology Co., Ltd.) according to the manufacturer's protocol. qPCR analysis was performed with SYBR Green I Mastermix (Roche Diagnostics GmbH, Mannheim, Germany) using a LightCycler 480 (Roche Diagnostics, Basel, Switzerland). The total reaction volume was $10 \mu \mathrm{l}$ which consisted of $0.25 \mu \mathrm{M}$ each primer and approximately $5 \mathrm{ng}$ of template cDNA. Accordingly, the thermocycling profiles were as follows: Denaturation at $95^{\circ} \mathrm{C}$ for $5 \mathrm{~min} ; 45$ cycles of $95^{\circ} \mathrm{C}$, $60^{\circ} \mathrm{C}$ for $20 \mathrm{sec}$ and $72^{\circ} \mathrm{C}$ for $20 \mathrm{sec}$, the melt curve protocol was $95^{\circ} \mathrm{C}$ for $5 \mathrm{sec}, 65^{\circ} \mathrm{C}$ for $1 \mathrm{~min}$ and reheating to $97^{\circ} \mathrm{C}$. Finally, the temperature was reduced to $40^{\circ} \mathrm{C}$ and maintained for $10 \mathrm{sec}$. The specific primers for miR-93 was synthesized as forward 5'-AGTCTCTGGCTGACTACATCACAG-3' and reverse 5'-CTACTCACAAAACAGGAGTGGAATC-3'. U6 (forward 5'-CTCGCTTCGGCAGCACA-3' and reverse 5'-AACGCT TCACGAATTTGCGT-3') was used as the internal control. Data were analyzed using the $2^{-\Delta \Delta \mathrm{Cq}}$ method (16).

Western blot analysis. The online tool microRNA.org (www.microrna.org/microrna/home.do) was used to predict the target gene for miR-93, and was used to select the specific primary antibody for the present study. Protein samples of the infected GTM cells were prepared using RIPA lysis buffer (Beyotime Institute of Biotechnology, Inc., Shanghai, China). The protein contents were quantified using the BCA Protein Assay kit (Pierce; Thermo Fisher Scientific, Inc.). A protein sample of $20 \mu \mathrm{g}$ was loaded in each lane for separation of the samples by $12 \%$ sodium dodecyl sulfate-polyacrylamide gel electrophoresis, following which they were transferred onto a polyvinylidene fluoride membrane. The membrane was blocked in 5\% skim milk for $2 \mathrm{~h}$ at room temperature and incubated with anti-NFE2L2 (monoclonal; rabbit anti-human; 1:1,000; cat. no. ab62352) or anti-GAPDH (internal reference; monoclonal; rabbit anti-human; 1:10,000; cat. no. ab181602) primary antibodies (Abcam, Cambridge, UK) at $4^{\circ} \mathrm{C}$ overnight. Following washing with a buffer containing 0.1\% Tween-20, 20 $\mathrm{mM}$ Tris-HC and $137 \mathrm{mM} \mathrm{NaCl}$ (TBST), the membrane was incubated in horseradish peroxidase-conjugated secondary antibody (polyclonal; goat anti-rabbit; 1:10,000; cat. no. ab97051) for $1 \mathrm{~h}$ at room temperature. Signals were developed using ECL Plus western blotting substrate (Thermo Fisher Scientific, Inc.) and the intensity of bands was analyzed using the ChemiDoc XRS system (Bio-Rad Laboratories, Inc., Hercules, CA, USA).

Statistical analysis. All experiments were repeated in triplicate, and results are presented as the mean \pm standard deviation. Statistical analysis was performed using SPSS 19.0 software (IBM SPSS, Armonk, NY, USA) using one-way analysis of variance. $\mathrm{P}<0.05$ was considered to indicate a statistically significant difference.

\section{Results}

miR-93 is upregulated in GTM cells. Prior to investigating the functions of miR-93, the expression levels of miR-93 in 


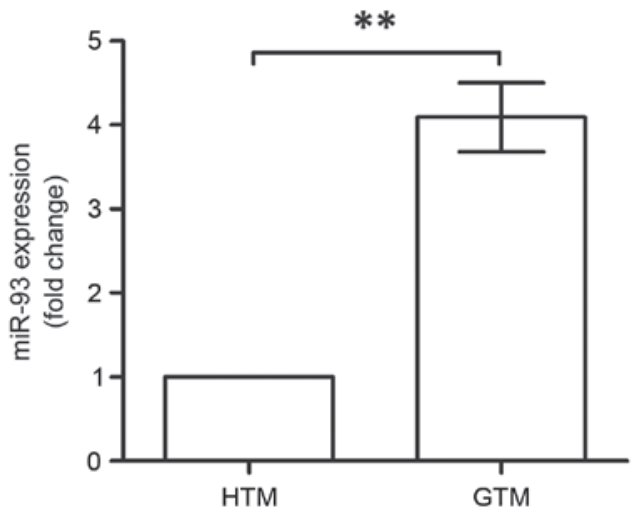

Figure 1. miR-93 is upregulated in GTM cells. The expression of miR-93 was significantly higher in the GTM cells, compared with the HTM cells (** $\mathrm{P}<0.01)$. miR-93, microRNA-93. HTM, human trabecular meshwork; GTM, glaucoma trabecular meshwork.
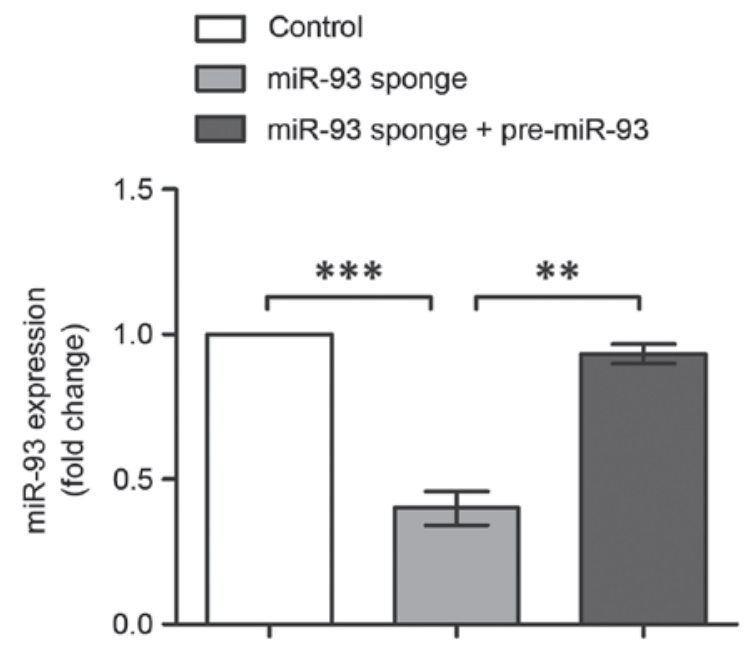

Figure 2. Expression of miR-93 in GTM cells with inhibition or overexpression of miR-93. The expression of miR-93 in GTM cells was inhibited by transfection with its inhibitor sponge $\left({ }^{* * *} \mathrm{P}<0.001\right)$. When GTM cells were co-transfected with the miR-93 sponge and pre-miR-93 vector, the effects of the miR-93 sponge were abrogated by the overexpression of miR-93 $\left({ }^{* *} \mathrm{P}<0.01\right)$. miR-93, microRNA-93; pre-miR-93, miR-93 precursor; GTM, glaucoma trabecular meshwork.

HTM and GTM cells were compared using RT-qPCR analysis (Fig. 1). The expression of miR-93 was significantly upregulated in the GTM cells, which was almost four times higher, compared with that in the HTM cells $(\mathrm{P}<0.01)$. This indicated the association between miR-93 and glaucoma; therefore, it was necessary to reveal the roles of miR-93 in GTM.

miR-93 inhibits viability and promotes apoptosis of GTM cells. To investigate the role of miR-93 in GTM cells, miR-93 was inhibited and overexpressed by transfection with its specific inhibitor sponge and expression vector, respectively. Alterations in the expression of miR-93 were analyzed using RT-qPCR analysis (Fig. 2). The results indicated the successful inhibition and overexpression of miR-93, with its expression levels significantly reduced following miR-93 sponge transfection $(\mathrm{P}<0.001)$, and recovered to the original level following co-transfection with the miR-93 sponge and pre-miR-93 vector $(\mathrm{P}<0.01)$. Therefore, these three groups of transfected cells were suitable for use in the following experiments.

GTM cell viability was monitored during the $96 \mathrm{~h}$ time period following transfection (Fig. 3A). The viability of the GTM cells in the control group gradually decreased from $48 \mathrm{~h}$ post-transfection. By contrast, the cells with inhibited miR-93 showed marked promotion of cell viability, which was significant higher, compared with that in the control group $(\mathrm{P}<0.01)$. The results also showed that cell viability was inhibited when miR-93 was overexpressed $(\mathrm{P}<0.05)$, which indicated that miR-93 was capable of inhibiting GTM cell viability. At $96 \mathrm{~h}$ post-transfection, the percentages of apoptotic cells were also examined using annexin V-FITC/PI staining (Fig. 3B). Compared with the control group, cell apoptosis was inhibited when miR-93 was suppressed $(\mathrm{P}<0.001)$. However, in the cells overexpressing miR-93, the effects of the miR-93 sponge was abrogated, with the percentage of apoptotic cells significantly increased $(\mathrm{P}<0.001)$, and an increase in cells in the late stage of apoptosis. Taken together, these results indicated that miR-93 induced the apoptosis of GTM cells and inhibited their viability, suggesting it is an important regulator in glaucoma.

miR-93 suppresses the expression of NFE2L2. The present study subsequently investigated the regulatory mechanism of miR-93. As a previous study reported that NFE2L2 is a target gene of miR-93 during breast carcinogenesis (17), the protein expression levels of NFE2L2 were analyzed in the transfected GTM cell groups in the present study (Fig. 4). The results indicated that the expression of NFE2L2 was promoted in the GTM cells with inhibited miR-93 $(\mathrm{P}<0.01)$ and was suppressed when miR-93 was overexpressed in the cells $(\mathrm{P}<0.01)$. These results suggested that miR-93 inhibited NFE2L2 in the GTM cells, which may be the possible functional pathway underlying the effect of miR-93 on regulating glaucoma.

\section{Discussion}

In the present study, miR-93 was found to be upregulated in GTM cells. The inhibition of miR-93 resulted in the promotion of cell viability and suppression of cell apoptosis, whereas the overexpression of miR-93 abrogated these effects. miR-93 was further found to suppress the expression of NFE2L2, which provides a possible clue to the functional mechanism of miR-93 in regulating GTM cells.

NFE2L2 is a regulator mediating the transcription process of cytoprotective genes, which has been well-characterized in previous studies of oxidative stress. Oxidative stress, together with mitochondria impairment and pathogenic events, contributes to a complex network of mechanisms leading to glaucoma $(18,19)$. Under quiescent conditions, NFE2L2 localizes in the cytoplasm anchored by Kelch ECH associating protein 1 (KEAP1). When the cells are stimulated by chemical signals, NFE2L2 escapes from KEAP1 and is translocated to the nucleus, where it activates the expression of target genes to enhance cell survival (20). To be specific, NFE2L2 prevents chromium (VI)-induced cell apoptosis and oxidative stress via promoting the expression of cytoprotective genes, including heme oxygenase 1 and $\mathrm{NAD}(\mathrm{P}) \mathrm{H}$ : quinone oxidoreductase 1 (21). It also assists in resistance against nitric oxide-dependent toxicity, thus preventing motor 
A

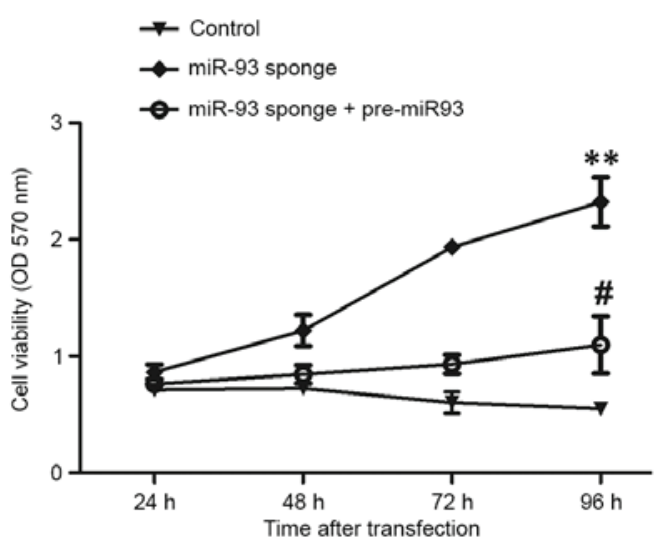

B

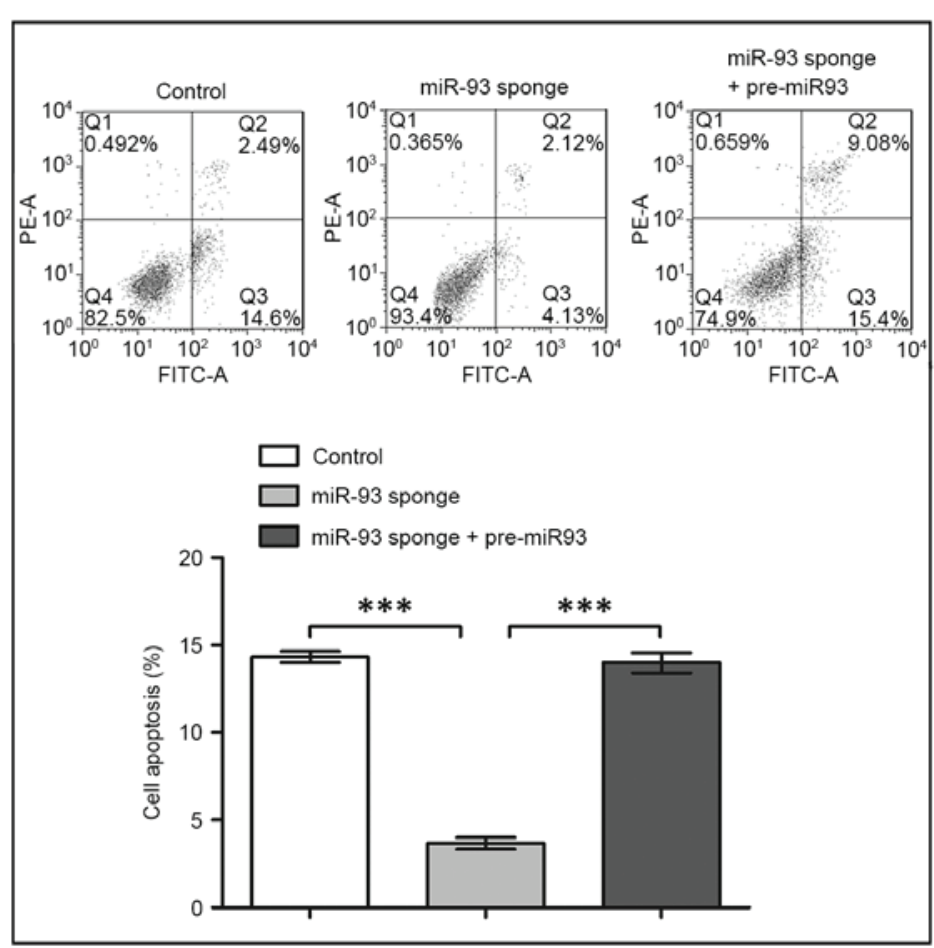

Figure 3. Inhibition of miR-93 leads to the promotion of cell viability and suppression of apoptosis in GTM cells. (A) Cell viability indicated by the OD at $570 \mathrm{~nm}$ was monitored following the transfection of GTM cells with miR-93 sponge and pre-miR-93 for 24, 48,72 and 96 h. At 96 h post-transfection, the inhibition of miR-93 led to a significant increase in cell viability, compared with the control group $\left({ }^{* *} \mathrm{P}<0.01\right)$. At $96 \mathrm{~h}$ post-transfection, the viability of cells co-transfected with the miR-93 sponge and pre-miR-93 were significantly decreased, compared with those transfected with the miR-93 sponge alone $\left({ }^{\#} \mathrm{P}<0.05\right)$. (B) GTM cell apoptosis was inhibited by suppressing miR-93 and promoted by co-transfection with pre-miR-93. The histogram shows the percentages of apoptotic cells in Q3 (*** $<<0.001)$. miR-93, microRNA-93; pre-miR-93, miR-93 precursor; GTM, glaucoma trabecular meshwork; OD, optical density; PE, phycoerythrin; FITC, fluorescein isothiocyanate.

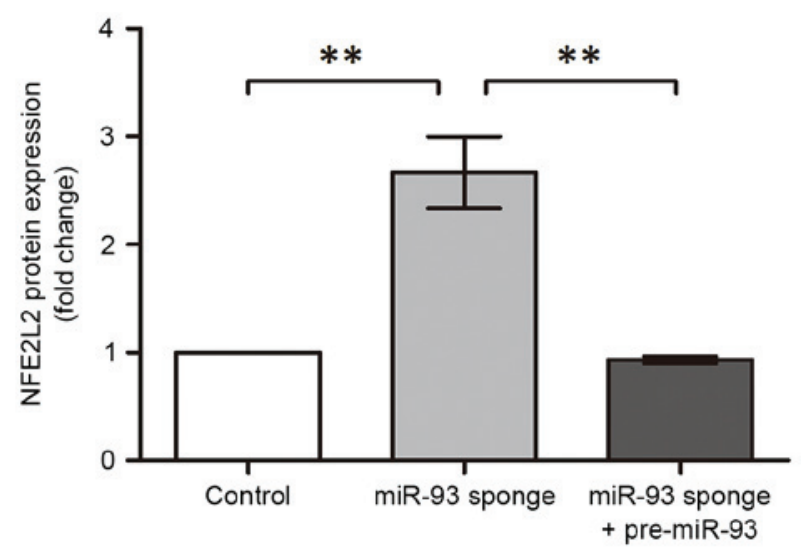

NFE2L2

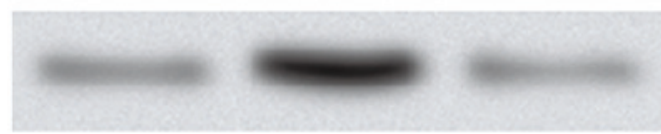

GAPDH

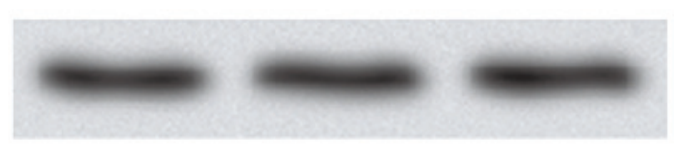

Figure 4. Protein expression of NFE2L2 is upregulated following the inhibition of miR-93, and this promotion is reversed by co-transfection with pre-miR-93. Western blot analysis was used to determine the protein expression levels of NFE2L2. GAPDH was used as the internal reference. The histogram shows the results from three replicates $\left({ }^{* *} \mathrm{P}<0.01\right)$. NFE2L2, nuclear factor, erythroid 2-like 2; miR-93, microRNA-93; pre-miR-93, miR-93 precursor. neuron apoptosis (22). In tumor cells, NFE2L2 functions as an inhibitor of apoptosis, with its silencing leading to the suppression of tumor growth (23). In the present study, the level of NFE2L2 was promoted when miR-93 was inhibited, which was accompanied by reduced GTM cell apoptosis. This result is concordant with those of existing reports that NFE2L2 acts as an anti-apoptotic factor (24-26), and suggested that NFE2L2 was involved in the regulation of GTM cell apoptosis.

As mentioned above, miRNAs execute their functions via regulating target genes post-transcriptionally. miR-93 can regulate vascular endothelial growth factor (VEGF) (27) and the phosphatase and tensin homolog/Akt pathway (28), thus modulating cell performance and disease progression. It also inhibits tumor growth in human colorectal cancer by suppressing the cell cycle-associated factor, cyclin B1, and cell proliferation-associated factors, Erb-B2 receptor tyrosine kinase 2, cyclin-dependent kinase inhibitor $1 \mathrm{~A}$ and VEGF (29). In the present study, miR-93 was found to inhibit the protein expression of NFE2L2. Although miR-93 was predicted to target the sequence 'CACUUU' of NFE2L2 using the online tool, microRNA.org (data not shown), whether NFE2L2 is directly bound and regulated by miR-93 remains to be elucidated. It is possible that other modulators regulated by miR-93 caused the observed downregulation of NFE2L2, which requires further investigation. However, based on the results of the present study, it was clear that miR-93 did function in GTM cells via regulating NFE2L2. The affected 
NFE2L2 may have inhibited the expression of its target genes, which contributed to the effects of miR-93 as a promoter of cell apoptosis in the GTM cells.

The dynamic and aberrant expression of miRNAs has been observed in various types of disease. The miR-23b/27b/24-1 cluster is downregulated in prostate cancer tissues, which can serve as a marker of progression and a tumor suppressor of this specific disease (30). miR-93 is dynamically expressed during neural stem cell differentiation (31). Similarly, in the present study, miR-93 was found to be upregulated in GTM cells, which was associated with its regulation of GTM cells by inhibiting cell viability and promoting cell apoptosis, possibly by suppressing the expression of NFE2L2. These specific expression profiles may provide tools for cell isolation, target gene modulation in specific stages and cell types, and gene therapy (32). As for glaucoma, gene therapy is promising owing to the modification and improvement of transgene vector delivery methods, including canaloplasty, which are under investigation $(33,34)$. Further investigations on the therapeutic targets of glaucoma are essential for the future implementation of gene therapy for glaucoma. It appears that miR-93 and its possible target, NFE2L2, discussed in the present study are significant as potential therapeutic targets for treating glaucoma.

In conclusion, the upregulation of miR-93 in GTM cells was associated with its regulatory functions on inhibiting cell viability and inducing cell apoptosis, which were possibly enabled by suppressing NFE2L2. These results elucidated the roles of miR-93 and its regulatory mechanism in GTM cells, providing fundamental information and potential therapeutic targets for treating glaucoma.

\section{References}

1. Kingman S: Glaucoma is second leading cause of blindness globally. Bull World Health Organ 82: 887-888, 2004.

2. Quigley HA: Glaucoma. Lancet 377: 1367-1377, 2011.

3. Gasiorowski JZ and Russell P: Biological properties of trabecular meshwork cells. Exp Eye Res 88: 671-675, 2009.

4. Mozaffarieh M, Grieshaber MC and Flammer J: Oxygen and blood flow: Players in the pathogenesis of glaucoma. Mol Vis 14: 224-233, 2008.

5. Tektas OY and Lütjen-Drecoll E: Structural changes of the trabecular meshwork in different kinds of glaucoma. Exp Eye Res 88: 769-775, 2009.

6. Hausser $\mathrm{J}$ and Zavolan M: Identification and consequences of miRNA-target interactions-beyond repression of gene expression. Nat Rev Genet 15: 599-612, 2014.

7. Asirvatham AJ, Magner WJ and Tomasi TB: miRNA regulation of cytokine genes. Cytokine 45: 58-69, 2009.

8. Wu L and Belasco JG: Let me count the ways: Mechanisms of gene regulation by miRNAs and siRNAs. Mol Cell 29: 1-7, 2008.

9. Georges M, Coppieters W and Charlier C: Polymorphic miRNA-mediated gene regulation: Contribution to phenotypic variation and disease. Curr Opin Genet Dev 17: 166-176, 2007.

10. Das J, Podder S and Ghosh TC: Insights into the miRNA regulations in human disease genes. BMC Genomics 15: 1010, 2014.

11. Luna C, Li G, Huang J, Qiu J, Wu J, Yuan F, Epstein DL and Gonzalez P: Regulation of trabecular meshwork cell contraction and intraocular pressure by miR-200c. PLoS One 7: e51688, 2012.

12. Li G, Luna C, Qiu J, Epstein DL and Gonzalez P: Alterations in microRNA expression in stress-induced cellular senescence. Mech Ageing Dev 130: 731-741, 2009.

13. Li G, Luna C, Qiu J, Epstein DL and Gonzalez P: Targeting of integrin betal and kinesin 2alpha by microRNA 183. J Biol Chem 285: 5461-5471, 2010.

14. Yu XF, Zou J, Bao ZJ and Dong J: miR-93 suppresses proliferation and colony formation of human colon cancer stem cells. World J Gastroenterol 17: 4711-4717, 2011.
15. Du L, Schageman JJ, Subauste MC, Saber B, Hammond SM, Prudkin L, Wistuba II, Ji L, Roth JA, Minna JD and Pertsemlidis A: miR-93, miR-98, and miR-197 regulate expression of tumor suppressor gene FUS1. Mol Cancer Res 7: 1234-1243, 2009.

16. Livak KJ and Schmittgen TD: Analysis of relative gene expression data using real-time quantitative PCR and the 2(-Delta Delta C(T)) Method. Methods 25: 402-408, 2001

17. Singh B, Ronghe AM, Chatterjee A, Bhat NK and Bhat HK: MicroRNA-93 regulates NRF2 expression and is associated with breast carcinogenesis. Carcinogenesis 34: 1165-1172, 2013.

18. Izzotti A, Saccà SC,Longobardi M and Cartiglia C: Mitochondrial damage in the trabecular meshwork of patients with glaucoma. Arch Ophthalmol 128: 724-730, 2010.

19. Saccà SC and Izzotti A: Focus on molecular events in the anterior chamber leading to glaucoma. Cell Mol Life Sci 71: 2197-2218, 2014.

20. Kensler TW, Wakabayashi N and Biswal S: Cell survival responses to environmental stresses via the Keap1-Nrf2-ARE pathway. Annu Rev Pharmacol Toxicol 47: 89-116, 2007.

21. He X, Lin GX, Chen MG, Zhang JX and Ma Q: Protection against chromium (VI)-induced oxidative stress and apoptosis by Nrf2. Recruiting Nrf2 into the nucleus and disrupting the nuclear Nrf2/Keap1 association. Toxicol Sci 98: 298-309, 2007.

22. Vargas MR, Pehar M, Cassina P, Beckman JS and Barbeito L: Increased glutathione biosynthesis by $\mathrm{Nrf} 2$ activation in astrocytes prevents p75NTR-dependent motor neuron apoptosis. J Neurochem 97: 687-696, 2006.

23. Singh A, Boldin-Adamsky S, Thimmulappa RK, Rath SK, Ashush H, Coulter J, Blackford A, Goodman SN, Bunz F, Watson WH, et al: RNAi-mediated silencing of nuclear factor erythroid-2-related factor 2 gene expression in non-small cell lung cancer inhibits tumor growth and increases efficacy of chemotherapy. Cancer Res 68: 7975-7984, 2008.

24. Narasimhan M, Mahimainathan L, Rathinam ML, Riar AK and Henderson GI: Overexpression of Nrf2 protects cerebral cortical neurons from ethanol-induced apoptotic death. Mol Pharmacol 80: 988-999, 2011.

25. Son YO, Pratheeshkumar P, Roy RV, Hitron JA, Wang L, Zhang Z and Shi X: Nrf2/p62 signaling in apoptosis resistance and its role in cadmium-induced carcinogenesis. J Biol Chem 289: 28660-28675, 2014.

26. Bhakkiyalakshmi E, Shalini D, Sekar TV, Rajaguru P, Paulmurugan R and Ramkumar KM: Therapeutic potential of pterostilbene against pancreatic beta-cell apoptosis mediated through Nrf2. Br JPharmacol 171: 1747-1757, 2014.

27. Long J, Wang Y, Wang W, Chang BH and Danesh FR: Identification of microRNA-93 as a novel regulator of vascular endothelial growth factor in hyperglycemic conditions. J Biol Chem 285: 23457-23465, 2010.

28. Fu X, Tian J, Zhang L, Chen Y and Hao Q: Involvement of microRNA-93, a new regulator of PTEN/Akt signaling pathway, in regulation of chemotherapeutic drug cisplatin chemosensitivity in ovarian cancer cells. FEBS Lett 586: 1279-1286, 2012.

29. Yang IP, Tsai HL, Hou MF, Chen KC, Tsai PC, Huang SW, Chou WW, Wang JY and Juo SH: MicroRNA-93 inhibits tumor growth and early relapse of human colorectal cancer by affecting genes involved in the cell cycle. Carcinogenesis 33: 1522-1530, 2012.

30. Goto Y, Kojima S, Nishikawa R, Enokida H, Chiyomaru T, Kinoshita T, Nakagawa M, Naya Y, Ichikawa T and Seki N: The microRNA-23b/27b/24-1 cluster is a disease progression marker and tumor suppressor in prostate cancer. Oncotarget 5: 7748-7759, 2014.

31. Lattanzi A, Gentner B, Corno D, Di Tomaso T, Mestdagh P, Speleman F, Naldini L and Gritti A: Dynamic activity of miR-125b and miR-93 during murine neural stem cell differentiation in vitro and in the Subventricular Zone Neurogenic Niche. PLoS One 8: e67411, 2013.

32. Gentner B, Visigalli I, Hiramatsu H, Lechman E, Ungari S, Giustacchini A, Schira G, Amendola M, Quattrini A, Martino S, et al: Identification of hematopoietic stem cell-specific miRNAs enables gene therapy of globoid cell leukodystrophy. Sci Transl Med 2: 58ra84, 2010.

33. Tian B and Kaufman PL: A potential application of canaloplasty in glaucoma gene therapy. Transl Vis Sci Technol 2: pii: 2, 2013.

34. Aktas Z, Tian B, McDonald J, Yamamato R, Larsen C, Kiland J, Kaufman PL and Rasmussen CA: Application of canaloplasty in glaucoma gene therapy: Where are we? J Ocul Pharmacol Ther 30: 277-282, 2014. 